\title{
La lectura de cuentos dialógica y las características de la interacción según estrato
}

\section{social de procedencia.}

\author{
Telma Piacente ${ }^{1} \&$ Ana María Tittarelli \\ Universidad Nacional de La Plata, Facultad de Psicología \\ Ciudad de a Plata, Provincia de Buenos Aires, Argentina
}

\begin{abstract}
Resumen. El propósito de este estudio descriptivo realizado en el Gran La Plata (Argentina) fue evaluar la disponibilidad de recursos que favorecen la lectura y las características de la interacción en la lectura de cuentos destinada a niños, según estrato social de procedencia. Los datos fueron recogidos de una muestra intencional de madres alfabetizadas, 50 de estrato social bajo y 50 de estrato social medio-bajo. Se utilizó una entrevista semiestructurada y precodificada, para examinar distintos aspectos de los contextos hogareños sobre las áreas mencionadas. Las respuestas fueron clasificadas en tres categorías: adecuada, intermedia, necesita mejorar, según la presencia, oportunidad, frecuencia e intensidad de los aspectos indagados. Los resultados obtenidos muestran diferencias significativas entre ambos grupos y diferencias intragrupales. Se presentan reflexiones sobre los alcances y limitaciones del presente estudio, que pueden orientar futuras investigaciones. Finalmente se destaca la importancia de la promoción de la lectura dialógica de cuentos en los años preescolares.
\end{abstract}

Palabras clave: Cuentos, Lectura, Interacción, Nivel socioeconómico.

Title: The dialogic reading of story and the characteristics of the interaction according to the readers' social class.

Abstract: The aim of this descriptive study, which was carried out in the Gran La Plata region in Argentina, was to evaluate the availability of resources that favour reading and the characteristics of the interaction in the reading of story to children, with consideration of the social group the subjects belonged to. The data comes from an intentional sample of literate mothers, 50 from a low social class and 50 from a medium to low social class. A semistructured and precodified interview was used to examine the different aspects of the home environments in the above mentioned areas. The answers were classified according to the presence, opportunity, frequency and intensity of the investigated aspects in three categories: 'adequate', 'intermediate' and 'it needs improvement'. The results obtained show significant intergroup and intragroup differences. Reflections on the scope and limitations of the present study are presented in the belief that they can serve as a starting point for future research. In addition, the importance of a dialogic reading of story in pre-school stages is highlighted.

Key Words: Story, Reading, Interaction, Social Class.

\section{Introducción.}

El propósito del estudio que se presenta, realizado en el Gran La Plata (Argentina) fue evaluar la disponibilidad de recursos que favorecen la lectura y las características de la interacción en la lectura de cuentos destinada a niños, según estrato social de procedencia.

En la literatura especializada se ha destacado la importancia de la lectura interactiva de cuentos en los años preescolares, como uno de los tópicos de mayor interés para la alfabetización posterior. Uno de los debates tradicionales en Psicología y Pedagogía corresponde a la identificación del momento en el que iniciar la alfabetización. Los cambios

\footnotetext{
${ }^{1}$ Por favor dirigir la correspondencia relacionada con este artículo a:

Telma Piacente, E-mail: piacente@isis.unlp.edu.ar, TE: 5402214829693
} 
terminológicos que se han operado, particularmente en las últimas décadas dan cuenta no sólo del estado de las controversias sino, además, de las diferencias en la teoría, la investigación y la práctica educativa. Efectivamente cuando se precisan nociones tales como prelectura $\mathrm{o}$ preparación para la lectura no se afirma lo mismo que cuando se alude a la alfabetización emergente o alfabetización temprana. La delimitación del alcance de todas ellas supone hacer un recorrido, aunque breve, sobre la investigación en lectura de las últimas décadas y sobre las condiciones que posibilitan un aprendizaje exitoso.

El Siglo XX ha sido el escenario del estudio del lenguaje, en sus diversas manifestaciones y niveles de análisis. Particularmente interesantes han sido las indagaciones sobre las relaciones entre el lenguaje oral y escrito $\mathrm{y}$, en este último caso respecto de las demandas cognitivas referidas al aprendizaje de la lectura y escritura según sistemas de escritura particulares. Para el caso del español, ha cobrado relevancia la identificación de las habilidades y conocimientos implicados en el reconocimiento y escritura de palabras como condición de posibilidad para los procesos de nivel superior referidos a la comprensión y producción textual.

Paralelamente, y no siempre de modo sincrónico, las planificaciones tendientes a optimizar el proceso de alfabetización, han partido de modelos diferentes. Especialmente destacable es el hecho de que en algunos de ellos, el supuesto acerca de aquello que constituye aprender a leer y a escribir, se inscribe en consideraciones referidas al nivel mental de los niños o bien a un conjunto de habilidades perceptuales y psicomotrices. Frente a esto la investigación cognitiva y psicolingüística ha enfatizado la importancia de los procesos cognitivos de dominio específico, así como de los conocimientos necesarios para tales aprendizajes. En tal sentido se especifican las habilidades de conciencia fonológica, que posibilitan la segmentación de la sustancia fónica en sus elementos constituyentes (los fonemas), el conocimiento del mundo de lo escrito, particularmente el conocimiento de los rasgos distintivos de las letras y las habilidades de poner en correspondencia fonemas y grafemas. Dicho de otro modo, la necesidad de dominar el principio alfabético para poder leer y escribir en un sistema de escritura alfabético.

Naturalmente la finalidad última de la alfabetización en una cultura letrada es habilitar a los alumnos para que puedan interactuar con textos complejos, tanto en producción como en comprensión. Pero el camino a recorrer es amplio y comienza en los primeros momentos de la vida. En esta perspectiva, ha cobrado importancia la consideración de la alfabetización 
emergente posteriormente reconceptualizada como alfabetización temprana. La primera alude al conjunto de habilidades y conocimientos que emergen a partir del contacto del niño preescolar con un medio rico en experiencias de lectura y escritura. Si bien esa noción se adecua descriptivamente a las características contextuales que posibilitan el desarrollo de ciertas habilidades y conocimientos de parte de los niños, no da cuenta de las características interaccionales que suceden en ese contexto. En tal sentido los términos alfabetización temprana delimitan los esfuerzos parentales y del personal de servicios de atención al preescolar por enseñar de manera explícita aspectos significativos relativos al lenguaje escrito.

Entre las indagaciones a propósito de las interacciones adulto-niño en torno al lenguaje escrito, se han destacado los estudios sobre la lectura interactiva. En ellos es posible observar distintos focos de interés, en la medida que algunos están dirigidos a contrastar resultados, otros se han centrado en procesos y finalmente algunos en la naturaleza evolutiva de las manifestaciones examinadas.

El propósito general de las investigaciones basadas en resultados es examinar las relaciones entre las variables de alfabetización temprana y aquellas consideradas más representativas de la alfabetización convencional. Tales indagaciones se conducen usualmente de acuerdo a diseños cuasi experimentales.

Las investigaciones basadas en procesos, que tienden a adherir a principios más cualitativos o interpretativos (Ericsson, 1990), enfatizan la marcha simultánea de los inputs en la experiencia de alfabetización infantil y tienden a utilizar términos como transacción, reorganización y mediación para describir la relación entre los numerosos y multifacéticos estratos de los procesos socioculturales y cognitivos involucrados en el crecimiento de la lectura y escritura en los niños pequeños.

Finalmente las investigaciones evolutivas, también basadas en enfoques interpretativistas, destacan la evolución "momento a momento", la naturaleza sistemática del crecimiento en alfabetización. Las descripciones de estas investigaciones están orientadas a discernir el efecto de la alfabetización temprana sobre la alfabetización posterior. 


\section{El papel de la lectura interactiva.}

Ahora bien, más allá del interés específico de cada una de las orientaciones de esas investigaciones, en todas se señala la importancia de la lectura interactiva. Su diferencia con otros modos de lectura radica en que incluye conversaciones, que implican dar lugar a diferentes turnos en el diálogo y que involucran activamente a los niños en ese proceso, conduciéndolos progresivamente de mundos actuales a mundos posibles (DeBruin-Perecki, 2004).

Dado que los libros constituyen "el alma y el corazón de la lectura" (Graves, Juel \& Graves, 2001, p.90), aquello que se lee a los niños a menudo constituye la reflexión de padres y cuidadores sobre la clase de libros a los que pueden estar expuestos. Los libros que son releídos se transforman en libros respondidos con entusiasmo y los niños solicitan insistentemente que se les vuelvan a leer.

Los niños aprenden acerca del mundo, en gran medida a través de los libros, ya que por su intermedio se transmiten valores, creencias, tradiciones, conocimientos y lenguaje. Pero además los niños aprenden mucho acerca de los libros y de cómo se lee a partir de los libros que se les leen.

Cuando se analizan las características de la lectura interactiva, se observa que sirve a propósitos múltiples, a saber: (1) Promover una activa participación de los niños en la construcción de la comprensión del significado de un libro, posibilitando la realización de inferencias, arribar a conclusiones y hacer predicciones. (2) Esclarecer la comprensión infantil del texto por medio de una cognición compartida. (3) Expandir la comprensión infantil del significado de las palabras. (4) Promover el uso del vocabulario nuevo proporcionado por el texto en conversaciones acerca de aquello que sucede en el libro y en la vida. (5) Familiarizar a los niños con conceptos relacionados con el mundo de lo escrito y con su tipo particular de registro. (6) Ayudar a los niños a ser más conscientes y a atender a la variedad de sonidos incluidos en las palabras que riman. (7) Promover oportunidades para la identificación de letras y sonidos. (8) Motivar a los niños para desarrollar el amor por los libros y el deseo de leer.

La lectura interactiva ocurre en una variedad de escenarios, tales como el hogar y el Jardín de Infantes, en una multiplicidad de ocasiones. Se implementa a través de una variedad de lectores, en situaciones en las que están incluidos padres y niños, abuelos/abuelas y nietos, maestros y alumnos preescolares, entre otros. Los propósitos instruccionales, explícitos o 
implícitos que conllevan, no dejan de lado su calidad de actividad entretenida y divertida. No obstante, para cumplir con los primeros es importante identificar no sólo la presencia y frecuencia de lectura de cuentos sino además conocer el modo en el que se lee. Efectivamente la lectura interactiva puede ser comprometida e instruccional sin perder por eso su carácter placentero, de modo tal de favorecer no sólo las habilidades y conocimientos en torno al lenguaje escrito sino además el placer de hacerlo, particularmente de modo compartido.

\section{Importancia de los "estilos" en la lectura de cuentos y de las características textuales.}

El valor de la lectura interactiva, reside entre otras razones en que implica a la vez los factores instrumentales que promueven habilidades de lectura y los factores motivacionales necesarios para convertirse en un lector efectivo. La investigación sobre las fuentes de variabilidad en la lectura de cuentos ha aumentado considerablemente en los últimos 10 años. Entre ellas se destacan las referidas a las conductas adultas y a la estructura del texto. Esos estudios tienden a orientarse sobre las categorías de "orientadas en procesos" o "evolutivas" porque sus objetivos son describir de manera más circunscripta la naturaleza de la interacción en sí misma, e inferir cuáles pueden ser los efectos a largo plazo de la lectura de cuentos.

Respecto de las características individuales de los padres y los niños, se ha examinado la frecuencia de lecturas compartidas. Entre las variables que inciden a ese respecto se ha encontrado que el nivel de relaciones emocionales seguras entre la madre y el niño está relacionado con los eventos de lectura en el hogar, de modo tal que esas experiencias pueden ser fuertemente dependientes de la historia parento filial. Asimismo, progresivamente se examinan las características de los formatos de interacción, particularmente durante la lectura de cuentos dialógica.

Unida a esas características aparecen las de la estructura del texto que se lee. Distintas investigaciones han examinado los efectos diferenciales que tienen la (a) organización de los textos (narrativos vs. expositivos); (b) los formatos de los textos, que incluyen por ejemplo ilustraciones o imágenes sobresalientes que pueden modificar la naturaleza de las interacciones entre padres y niños respecto de los aspectos gráficos, (c) la familiaridad con el texto debido a la influencia de la repetición de la lectura sobre la posibilidad de hacer inferencias y predicciones (Elster \& Walter 1992) y (d) la complejidad de la organización textual. 


\section{El andamiaje de la lectura interactiva.}

Aunque las prácticas de lectura conjunta de cuentos enriquecen las habilidades de lectura y comprensión, restan algunos interrogantes acerca de las características específicas de las sesiones interactivas que conduzcan a los niños a tener éxito con la lectura. En primer lugar es necesario volver a señalar que los niños incrementan no sólo la comprensión de lo leído a través del diálogo, sino además el conocimiento de las convenciones de la alfabetización, las características diferenciales del estilo de lo escrito y el placer por la lectura (Martin \& Reutzel, 1999). Para ello, el adulto debe ser capaz de juzgar el nivel de comprensión y conocimientos de los niños y la manera de conducirlo más allá. Dicho de otro modo, de la zona de desarrollo actual a la zona de desarrollo próximo (Vygotsky, 1978).

La diferencia entre la lectura interactiva y otros modos de presentar los libros radica justamente en las conductas de andamiaje (Whiterhurst \& Lonigan, 2003). La interacción que toma lugar en la primera requiere un cambio de roles de acuerdo a los cuales el padre/cuidador se transforma en un activo interlocutor que hace preguntas, adiciona información y conduce al niño para mayores elaboraciones sobre las ideas. Este tipo de estrategias transforma no sólo el vocabulario expresivo y comprensivo de los niños, sino también la complejidad gramatical del lenguaje infantil, el dominio semántico y el desarrollo de aspectos pragmáticos.

En investigaciones particulares se han identificado distintos tipos de estrategias interactivas (Morrow, 1990), que sucintamente pueden enumerarse de la siguiente manera: (1) Modelar la lectura. (2) Interrogar. (3) Andamiar el diálogo y las respuestas. (4) Ofrecer elogios o feedback positivo. (5) Proporcionar o extender la información. (6) Reformular información. (7) Dirigir la discusión. (8) Compartir reacciones personales. (9) Relacionar conceptos con las experiencias de la vida. (10) Gustar y disfrutar de la lectura. Las mismas pueden aparecer de modo singular o bien de modo complementario, combinándose en muchas ocasiones. Desde la perspectiva de la capacitación de los padres y/o maestros es necesario examinarlas y esclarecer sus alcances (Bergin, 2001; Hiebert, 1981; Holdaway, 1979).

\section{El impacto del medio en la lectura interactiva.}

Las habilidades y conocimientos prelectores que desarrollan los niños antes de llegar a la escuela no son algo que proviene del calendario madurativo. Es abundante el trabajo realizado respecto de la importancia que tienen en los aprendizajes posteriores (Dickinson \& 
Neuman, 2006). Particularmente en muchas investigaciones se ha señalado que los contextos de crianza no siempre proporcionan un ambiente rico en oportunidades de contacto con el lenguaje escrito. Para ello es menester disponer no sólo de adultos alfabetizados, cuyas prácticas de lectura y escritura sean variadas y frecuentes, de materiales de lectura y escritura, sino además, contar con hogares en los que se propicie que los niños participen en interacciones específicas con los cuidadores, que favorezcan aprendizajes tales como participar en lecturas dialógicas de cuentos, aprender el nombre de las letras, la escritura del nombre propio y otras palabras familiares, prácticas de escritura conjunta, entre otras (Stanovich, 1986, 2000; Teale, 1986).

En estudios nacionales e internacionales se han encontrado diferencias en torno a las interacciones lingüísticas materno filiales, a la disponibilidad de material impreso, a la frecuencia con que se les lee a los niños, al nivel educacional de los padres, entre otras variables, según estrato social de procedencia Payne, Whitehurst, \& Angell, 1994, Piacente, Marder, \& Resches, 2008).

En la mayoría de las investigaciones se han tomado en cuenta medidas del nivel socioeconómico (NSE). No obstante una literatura creciente sugiere que existen substanciales diferencias individuales en las prácticas hogareñas de familias de estratos bajos que pueden afectar el éxito escolar de los niños (Teale, 1986).

Heath $(1983,1986)$ en un amplio estudio etnográfico sobre los usos del lenguaje y la comunicación, analizó lo que ocurría específicamente durante la lectura de cuentos en distintas comunidades. Encontró que en las familias de estratos pobres era inexistente o escasa la práctica de lectura de cuentos. En las familias de estratos medios, si bien leían a sus hijos, aparecían dos modalidades diferentes. Mientras en un grupo se trataba de lectura interactiva, con generalizaciones a otros contextos, en el otro no, limitándose a la lectura ausente de interacción. Estas tres modalidades marcaron diferencias significativas en el rendimiento escolar posterior. En el primer caso aparecieron muchas dificultades. En el segundo la escolaridad no presentó problemas. En el último, los niños comenzaron a fracasar durante el tercer año, cuando se esperaba de ellos manifestaciones más creativas y conceptuales.

En investigaciones realizados en el Gran La Plata los resultados encontrados se inscriben en la misma dirección (Piacente, Marder, Resches \& Ledesma, 2008).

A continuación se presentan datos de una investigación más amplia, centrados en este 
caso en la presencia de lecturas compartidas y en las características que asumen según estrato social de procedencia.

\section{Las interacciones en lectura en familias de estratos bajos y medio bajo del gran la plata (provincia de buenos aires-argentina).}

En este trabajo se comunican exclusivamente los resultados encontrados referidos a las características de los contextos hogareños según se tratara de familias de estrato socioeconómico bajo y de estrato medio-bajo, respecto de los recursos y las prácticas familiares en torno a la lectura.

\subsection{Objetivos}

(1) Examinar la disponibilidad de recursos y prácticas de lectura que favorecen la alfabetización en familias de diferente procedencia sociocultural.

(2) Comparar las diferencias intra e intergrupales entre ambos grupos de familias.

\section{Material y Método}

\subsection{Participantes.}

Se seleccionó una muestra intencional de madres alfabetizadas, 50 de familias de estrato social bajo, beneficiarias del planes sociales (en adelante Grupo I) y 50 de un grupo de referencia de estrato social medio-bajo (en adelante Grupo II), que residían en el Gran La Plata, Provincia de Buenos Aires (Argentina).

El estrato social de procedencia fue identificado a partir del nivel educacional y la categoría ocupacional. En la Tabla 1 se ofrece información sobre los años de escolaridad de los padres de ambos grupos. El análisis de las diferencias de proporciones intergrupales arroja valores que alcanzan significación estadística (madre: Chi2: 53,805, p .000; padre: Chi2 48.896, p .000).

En cuanto a la categoría ocupacional, el $70 \%$ de las madres y el $65 \%$ de lo padres del GI no tienen un trabajo estable. Esa situación es diferente entre los padres del GII (10\% en el caso de las madres y $4,8 \%$ en el de los padres). Del total de la población activa, las madres del GI mayoritariamente realizan actividades de servicio doméstico y los padres son cuentapropistas. En el GII las madres y padres trabajan mayoritariamente como empleados. En la Tabla 2 se proporcionan los datos de las ocupaciones de ambos padres en los grupos estudiados 
Tabla 1.

Nivel educacional de los padres según años de escolaridad.

\begin{tabular}{lcccc}
\hline Indicadores & Madre GI & Madre G II & Padre GI & Padre GII \\
\hline 1-6-años & 16,5 & 2,1 & $19,8 \%$ & $5,0 \%$ \\
$7-9$ años & $59,2 \%$ & $25,5 \%$ & $59,4 \%$ & $35,0 \%$ \\
10 y más años & $24,3 \%$ & $72,4 \%$ & $20,8 \%$ & $60,0 \%$ \\
\hline
\end{tabular}

Nota: $p=.000$

Tabla 2.

Categoría ocupacional de los padres de ambos grupos

\begin{tabular}{lcccc}
\hline Indicadores & Madre GI & Madre G II & Padre GI & Padre GII \\
\hline Cuenta propia & $10,5 \%$ & $1,1 \%$ & $85,7 \%$ & $15,7 \%$ \\
Servicio doméstico & $69,2 \%$ & $4,5 \%$ & -- & -- \\
Empleado & $20,3 \%$ & $93,4 \%$ & $14,3 \%$ & $84,3 \%$ \\
\hline
\end{tabular}

\subsection{Instrumento.}

El instrumento utilizado fue una entrevista semiestructurada de elección múltiple (de 3 a 6 opciones), de 72 ítems, “Evaluación del Contexto Alfabetizador” (Piacente, Querejeta, Marder \& Resches, 2003). Se han seleccionado para el presente trabajo 7 ítems que focalizan las características de los hogares respecto de la disponibilidad de recursos, las prácticas la y modalidad interaccional de la lectura de cuentos, a saber:

1. Disponibilidad de material que favorece la alfabetización: (a) existencia y cantidad de libros en general y (b) existencia de libros destinados a los niños en particular.

2. Prácticas de lectura de cuentos. (a) edad de comienzo de la lectura de cuentos, (b) frecuencia de lectura de cuentos, (c) frecuencia de la solicitud infantil de lectura de cuentos, (d) disfrute infantil de la lectura de cuentos y (e) características de la interacción en la lectura de cuentos.

\subsection{Procedimiento.}

\subsubsection{Obtención y registro de los datos.}

Los cuidadores fueron entrevistados en sus hogares, por evaluadores especialmente entrenados. El registro de las respuestas fue supervisado por coordinadores expertos y volcados a una base de datos. 


\subsubsection{Análisis y elaboración de los datos.}

Las respuestas obtenidas se clasificaron en tres categorías, de menor a mayor adecuación, de acuerdo al criterio de presencia, oportunidad, frecuencia e intensidad de los aspectos indagados, según los ítems considerados. Las categorías resultantes fueron las siguientes.

A. Disponibilidad de libros

1. Necesita mejorar: presencia de 0-10 libros

2. Intermedio: presencia de 11-20 libros

3. Adecuado: presencia de 21 o más libros.

B. Edad de comienzo de lectura de cuentos

1. Necesita mejorar: ausencia de lectura o lectura a partir de los 4 años.

2. Intermedio: comienzo de la lectura de cuentos entre los 2 y 4 años.

3. Adecuado: comienzo de la lectura de cuentos antes de los 2 años.

C. Frecuencia de lectura de cuentos:

1. Necesita mejorar: casi nunca o una o dos veces por mes.

2. Intermedio: una o dos veces por semana

3. Adecuado: todos o casi todos los días.

D. Frecuencia de pedido de lectura de cuentos de parte de los niños:

1. Necesita mejorar: no pide, sólo una o dos veces por mes.

2. Intermedio: una o dos veces por semana

3. Adecuado: todos o casi todos los días.

E. Disfrute infantil de la lectura

1. Necesita mejorar: no disfruta.

2. Intermedio: disfruta poco

3. Adecuado: disfruta mucho

F. Características de la interacción durante la lectura de cuentos

1. Necesita mejorar: lectura sin interacción.

2. Intermedio: medianamente interactiva, solo responde a preguntas formuladas por el niño y formula pocas preguntas.

3. Adecuado: altamente interactiva, promueve la comprensión a través de preguntas, proporciona explicaciones y expansiones ante las preguntas infantiles, hace y solicita reformulaciones, vincula los eventos del libro con los de la vida cotidiana. 
Se calcularon los porcentajes de respuestas en cada categoría en cada uno de los grupos a los fines de su comparación y se hicieron análisis de diferencias de proporciones entre los grupos.

\section{Resultados}

Se observaron situaciones muy diferentes en familias de estratos bajos y de estratos medio-bajo en la mayoría de las variables examinadas

\subsection{Disponibilidad de libros en el hogar.}

La importancia en la disponibilidad de recursos tales como libros reside en que su presencia en el hogar constituye la condición de posibilidad para que se produzcan interacciones frecuentes con el lenguaje escrito. Debe tenerse en cuenta que coadyuva a esa posibilidad la presencia de padres alfabetizados, criterio de inclusión de los sujetos de la muestra en este trabajo.

Cuando además se trata de libros destinados a los niños como los libros de cuentos, aparece consecuentemente la preocupación hogareña por el contacto de los niños con textos especialmente destinados a ellos.

Son significativas las diferencias respecto de la disponibilidad de libros de lectura, particularmente de libros de cuentos en los grupos estudiados, en favor del GII. En el primer caso en las categorías "Necesita mejorar" y "Adecuada". En el segundo en la categoría "Intermedia y "Necesita Mejorar" (Tablas 3 y 4).

\section{Tabla 3.}

Disponibilidad de libros

\begin{tabular}{lccc}
\hline Categorías & GI & GII & $\mathrm{z}$ \\
\hline Necesita mejorar & $65,7 \% \%$ & $41,7 \%$ & $2.206^{*}$ \\
Intermedio & $13,2 \%$ & $14,6 \%$ & -0.087 \\
Adecuado & $21,1 \% \%$ & $43,7 \%$ & $2.201^{*}$ \\
\hline
\end{tabular}

Nota: $* p<.05$ 
Tabla 4.

Disponibilidad de libros infantiles

\begin{tabular}{lccc}
\hline Categorías & GI & GII & z \\
\hline Necesita mejorar & $84,2 \%$ & $50 \%$ & $3.427^{* *}$ \\
Intermedio & $9,1 \%$ & $29,2 \%$ & $2.300^{*}$ \\
Adecuado & $6,7 \%$ & $20,8 \%$ & 1.737 \\
\hline
\end{tabular}

Nota: $* p<.05, * * p<.01$

\subsection{Edad de comienzo de la lectura de cuentos.}

No resulta suficiente la presencia de libros en el hogar, sino conocer qué se hace con ellos y con los niños. Los resultados obtenidos sobre la lectura de cuentos permiten observar marcadas diferencias, particularmente relacionadas con la edad de comienzo para hacerlo. Las madres del grupo de la pobreza comenzaban mucho más tardíamente, observándose diferencias significativas en las Categorías "Necesita mejorar" y “Adecuada” (Tabla 5).

\section{Tabla 5.}

Edad comienzo lectura de cuentos

\begin{tabular}{lccc}
\hline Categorías & GI & GII & z \\
\hline Necesita mejorar & $30,3 \%$ & $10,4 \%$ & $2.223^{*}$ \\
Intermedio & $49,5 \%$ & $43,8 \%$ & 0.371 \\
Adecuado & $20,2 \%$ & $45,8 \%$ & $2.510^{*}$ \\
\hline
\end{tabular}

Nota: $* p<.05$,

\subsection{Frecuencia de lectura de cuentos}

En cuanto a la frecuencia de lectura que informan las madres, si bien los porcentajes de respuestas más adecuadas son mayores en el GII, no alcanzan significación estadística respecto de las respuestas que corresponden al GI. (Tabla 6).

\section{Tabla 6.}

Frecuencia lectura cuentos

\begin{tabular}{lccc}
\hline Categorías & GI & GII & z \\
\hline Necesita mejorar & $28,4 \%$ & $22,9 \%$ & 0.401 \\
Intermedio & $32,2 \%$ & $31,3 \%$ & -0.118 \\
Adecuado & $39,4 \%$ & $45,8 \%$ & 0.445 \\
\hline
\end{tabular}




\subsection{Demandas infantiles de lectura}

Respecto de las solicitudes infantiles de lectura, las diferencias en los porcentajes en ambos grupos no alcanzan significación estadística, aunque es mayor el porcentaje de respuestas adecuadas en el GII (Tabla 7).

\section{Tabla 7.}

Pedido infantil de lectura de cuentos

\begin{tabular}{lccc}
\hline Categorías & GI & GII & z \\
\hline Necesita mejorar & $25,1 \%$ & $16,6 \%$ & 0.800 \\
Intermedio & $25,6 \%$ & $31,3 \%$ & 0.410 \\
Adecuado & $49,3 \%$ & $52,1 \%$ & 0.080 \\
\hline
\end{tabular}

\subsection{Disfrute infantil de la lectura de cuentos}

Cuando se trata del disfrute de las actividades de lectura, los porcentajes de respuestas resultan mayoritariamente ajustados en la perspectiva de favorecer el desarrollo de habilidades y conocimientos prelectores, prácticamente sin diferencias entre ambos grupos (Tablas 8).

\section{Tabla 8.}

Disfrute infantil de lectura de cuentos

\begin{tabular}{lccc}
\hline Categorías & GI & GII & z \\
\hline Necesita mejorar & $0,5 \%$ & $0 \%$ & - \\
Intermedio & $7,7 \%$ & $4,2 \%$ & 0.317 \\
Adecuado & $91,8 \%$ & $95,8 \%$ & 0.415 \\
\hline
\end{tabular}

\subsection{Características de la interacción durante la lectura de cuentos}

Párrafo aparte merece el tipo de interacción durante la lectura de cuentos. Su importancia en tanto formato privilegiado para atraer la atención de los niños y promover el incremento de conocimiento sobre mundos posibles y la reflexión sobre las mismas características del lenguaje escrito, muestra marcadas diferencias entre los grupos considerados, en las categorías "Intermedia" y "Adecuada" (Tabla 9). 


\section{Tabla 9.}

Disfrute infantil de lectura de cuentos

\begin{tabular}{lccc}
\hline Categorías & GI & GII & $\mathrm{z}$ \\
\hline Necesita mejorar & $10 \%$ & $0,9 \%$ & -0.171 \\
Intermedio & $50,3 \%$ & $7,9 \% * *$ & $4.447^{* *}$ \\
Adecuado & $40,7 \%$ & $91,2 \% * *$ & $5.117^{* *}$ \\
\hline
\end{tabular}

Nota: $* \mathrm{p}<.05, * * \mathrm{p}<.01$

El análisis particularizado del tipo de respuestas proporcionadas por las madres del GI, más allá de los porcentajes diferenciales que se registraron, ilustra sobre la ausencia o insuficiencia de verdadera interacción o de interacción enriquecedora, en la medida que o bien sólo se leía el cuento o bien sólo se hacían algunas preguntas o se respondía a las eventuales preguntas de los niños.

\section{Conclusiones.}

La importancia del conjunto de recursos y prácticas que promueven el conocimiento y placer del lenguaje escrito, particularmente en el caso de la lectura interactiva de cuentos durante los primeros años, respecto de sus beneficios en la escolaridad posterior, parece estar suficientemente documentada por los trabajos de investigación realizados a ese respecto (Purcell-Gates, 2000). No obstante, las situaciones a las que hemos aludido no puede dejar de lado el hecho de que no es la lectura conjunta de los libros por sí misma la que marca fuertes diferencias en el desarrollo de la alfabetización infantil, sino más bien lo que sucede en la díada durante la lectura.

Los datos informados, aunque sucintos, evidencian diferencias entre los grupos examinados, con resultados más críticos en las familias de la pobreza, situación que coincide con la bibliografía especializada a este respecto. Pero al mismo tiempo ilustran acerca de una variabilidad intragrupal en ambos casos, que debe ser considerada en atención a dos cuestiones a ser tenidas en cuenta en los diseños de intervención. En el grupo de familias pobres, a pesar de las dificultades materiales que enfrentan, se observa la presencia de un porcentaje de prácticas adecuadas. En el otro grupo, con menores dificultades, la presencia (aunque más exigua), de un porcentaje de prácticas inadecuadas.

Una de las limitaciones de este estudio corresponde al sesgo de deseabilidad social que puede impregnar las respuestas. En esa perspectiva, más allá de la diversidad obtenida, 
resulta necesaria su intensificación a través de la observación directa de lo que realmente sucede en los hogares en las díadas de lectura, cuando realmente se efectivizan. Pero, por otro lado, si los porcentajes de respuestas en las familias del GI que se ubican en la categoría adecuada son mayores respecto de las prácticas que verdaderamente implementan o de las situaciones que se verifican en sus hogares, testimonian de manera indirecta el conocimiento de las madres sobre aquello que resulta beneficioso para los niños, circunstancia a ser considerada en los programas de intervención.

Las prácticas familiares varían en función de la significación que la alfabetización tiene para culturas y contextos diferentes (Rogers, 2001). La lectura interactiva, tal como ha sido señalada, que promueve el desarrollo de los conocimientos y habilidades necesarios para la futura escolarización, debe ser objeto de otros estudios en diferentes ámbitos de atención al preescolar, particularmente en los Jardines de Infantes.

Los resultados obtenidos (y los que provengan de otras investigaciones) pueden ser esclarecedoras para capacitar a los padres y maestros sobre las características de la lectura interactiva que resultan de mayor interés. Los estudios realizados a ese respecto muestran que las prácticas que redundan en favor de los conocimientos y habilidades prelectoras son aquellas que están destinadas a promover conocimientos y facilitar la comprensión de aspectos como los siguientes (DeBruin-Parecki, 1999, 2003, Piacente, Marder, Resches \& Ledesma, 2008)

(1) La interacción entre imágenes y texto, para identificarla y distinguir las fuentes diferenciales de información que proporcionan.

(2) La relación entre los eventos de la historia, para afianzar la comprensión del niño sobre el cuento y de las estructuras narrativas.

(3)La expansión de los conocimientos, de hechos y situaciones reales y ficticias así como de las características de los libros, tales como: (a) Las partes que lo conforman, (b) la direccionalidad de la escritura, (c) las diferencias imagen/escritura/letras/números, (d) el juego con sonidos, el conocimiento de las letras y de las palabras, (e) el tipo de registro que corresponde al lenguaje oral y escrito y el tipo textual propio de las narrativas, (f) el gusto y disfrute de la actividad de lectura, relacionando la historia leída con información relevante en la vida de los niños, animando las voces y los personajes, mostrando el entusiasmo que se pone en la lectura, $(\mathrm{g})$ el respecto por las competencias de los niños cuando se lee (VernonFeagan, Hammer, Miccio, \& Manlove, 2003). 
Muchas de estas características no se corresponden con la situación verificada para un porcentaje de los grupos examinados, particularmente en el caso del grupo más pobre. En esa población, los indicadores de repitencia, abandono o nivel de competencia en lectura y escritura que alcanzan aún aquellos que finalizan los estudios obligatorios, deben ser tenidos en cuenta para implementar estrategias preventivas desde edades tempranas (Snow,Burns, \& Griffin, 1998). En especial, en los servicios de atención al preescolar, particularmente en los jardines de infantes y en los primeros grados escolares, deben intensificarse los esfuerzos para optimizar las oportunidades de aprendizaje, con las que deben contar todos los niños.

La lectura interactiva de cuentos parece ser una de las vías posibles para lograrlo. No obstante, no se puede dejar de considerar que, por importante que resulte, no es la única estrategia de promoción de la alfabetización temprana. Existe otro conjunto de estrategias a ser implementadas, con mayor énfasis en los niños que provienen de sectores vulnerables, para que los habiliten en el dominio del sistema de escritura y del lenguaje escrito, de modo tal de promover un tránsito escolar exitoso. En uno u otro caso, el camino de la alfabetización debe comenzarse muy temprano, dejando de lado consideraciones acerca de prerrequisitos o condiciones madurativas. Efectivamente, excepto en casos patológicos excepcionales, todos los niños "nacen listos para aprender".

\section{Referencias.}

Bergin, C. (2001). The parent-child relationship during beginning reading. Journal of Literacy Research, 22 (4), 681-706.

DeBruin-Parecki, A. (1999). The Adult/Child Interactive Reading Inventory: An Assessment of joint story book reading skills (Tech. Rep. № 2-004). Ann Arbor, MI: Center for Improvement of Early Reading Achievement.

DeBruin-Parecki, A. (2003). Evaluating adult/child interactive reading skill. In A. DeBruin-Perecki \& B. Krol-Sinclair (Eds.), Family literacy: From theory to practice (pp. 282-302). Newark DE: International Reading Association.

DeBruin-Perecki, A. (2004). It's and open book! Using interactive reading to evaluate early literacy skills at home and at school. Paper presented ad the fits annual CIERA Summer Institute, Ann Arbor, MI.

Dickinson, D. K., \& Neuman, S. B. (2006). Handbook of Early Literacy Research. Volumen 2. New York: Guilford Press. 
Elster, C. \& Walter, C. A. (1992). Flexible scaffolds: Shared reading and rereading of storybooks in Head Start Classrooms. In C. K. Kinzer \& D. J. Leu (Eds.), Literacy Research and practice: View from many perspectives: Forty First Yearbook of the National Reading Conference (pp. 445-452). Chicago: National Reading Conference.

Ericsson, F. (1990). Qualitative methods. In M. Wittrock (Ed.), Research in teaching and learning (vol. 2, pp. 77-194). New York: McMillan.

Graves, M. F., Juel, C. \& Graves, B. (2000). Teaching Reading in the 21st. Century. Boston: Allyn and Bacon.

Heath, S. B. (1983). Ways with words: language, life, and work in communities and classrooms. Cambridge, United Kingdom: Cambridge University Press.

Heath, S. B. (1986). What no bedtime story means: Narrative skills at home and at school. In B. B. Schieffelin y E. Ochs (Eds.), Language, socialization across cultures (pp. 97-124). Cambridge, United Kingdom: Cambridge University Press.

Hiebert, E. H. (1981). Developmental patterns and interrelationships of preschool children's print awareness. Reading Research Quarterly, 16 (2), 236-260).

Holdaway, D. (1979). The Foundations of Literacy. Sydney: Ashton Scholastic.

Martin, L. E. \& Reutzel, R. D. (1999). Sharing books: examining how and why mothers deviate from print. Reading Research and Instruction, 39 (1), 39-69.

Morrow, L. M. (1990). Assessing children's understanding of story through there construction and reconstruction of narrative. In L. M. Morrow \& J. K. Smith (Eds.), Assessment for instruction in early literacy (pp. 110-133). Upper Saddle River, NJ: Prentice Hall.

Payne, A. C., Whitehurst, G. J., \& Angell, A. L. (1994). The Role of Home Literacy Environment in the Development of Language Ability in Preschool Children from Low-income Families. Early Childhood Research Quarterly 9, 427-440. State University of New York at Stony Brook.

Piacente, T. Querejeta, M., Marder, S. \& Resches, M. (2003). Evaluación del contexto alfabetizador. Comisión de Investigaciones científicas de la Provincia de Buenos Aires

Piacente, T., Marder, S., \& Resches, M. (2008). Condiciones del niño y la familia para la alfabetización. Comisión de Investigaciones Científicas de la Provincia de Buenos Aires.

Purcell-Gates, V. (2000). Family Literacy- En M. L. Michael, P. B. Moshental, P. D. Pearson, \& R. Barr (Eds). Handbook of Reading Research. Volumen III. Mahwah, NJ: Erlbaum.

Rogers, R. (2001). Family literacy and the mediation of cultural models. National Reading Conference Yearbook, 50, 96-114.

Snow, C.E., Burns, M. \& Griffin, P. (Eds.) (1998). Preventing reading difficulties in young children: Committee on Prevention of Reading Difficulties in Young Children, Commission on 
Behavioral and Social Sciences and Education. National Research Council, Washington, DC: National Academy Press.

Stanovich, K. E. (1986). Matthew effects in reading: Some consequences of individual differences in the acquisition of literacy. Reading Research Quarterly, 21, 360-407.

Stanovich, K. E. (2000). Progress in Understanding Reading. Scientific Foundations and New Frontiers. New York: The Guilford Press.

Teale, W.H. (1986). Home background and young children's literacy development. In W.H. Teale \& E. Sulzby, Emergent literacy: writing and reading. Norwood, NJ: Ablex.

Vernon-Feagan, L., Hammer, C. H., Miccio, A., \& Manlove, E. (2003). Early language and literacy skill in low-income African-American and Hispanic children. In S. B. Newman \& D. K. Dickinson (Eds.), Handbook of Early Literacy Research (pp. 192-210). New York: Guilford Press.

Vygotsky, L. (1978). Mind in Society: the development of psychological processes. Cambridge, MA: Harvard University Press.

Whitehurst, G. J. \& Lonigan Ch. L. (2003). Emergent Literacy: Development from Pre-readers to Readers. In S. Newman \& D. Dickinson (Eds.), Handbook of Early Literacy Development. New York: Guilford. 